\title{
FOURIER INEQUALITIES WITH NONRADIAL WEIGHTS
}

\author{
C. CARTON-LEBRUN
}

\begin{abstract}
Let $\mathscr{F} f(\gamma)=\int_{\mathbf{R}^{n}}\left(e^{-2 i \pi \gamma^{*} x}-1\right) f(x) d x, n>1$, and $u, v$ be nonnegative functions. Sufficient conditions are found in order that $\|\mathscr{F} f\|_{q, u} \leq$ $C\|f\|_{p, v}$ for all $f \in L_{v}^{p}\left(\mathbb{R}^{n}\right)$. Pointwise and norm approximations of $\mathscr{F} f$ are derived. Similar results are obtained when $u$ is replaced by a measure weight. In the case $v(x)=|x|^{n(p-1)}$, a counterexample is given which shows that no Fourier inequality can hold for all $f$ in $L_{c, 0}^{\infty}$. Spherical restriction theorems are established. Further conditions for the boundedness of $\mathscr{F}$ are discussed.
\end{abstract}

\section{INTRODUCTION}

Let $n>1$ and $\mathscr{F} f(\gamma)=\int_{\mathbb{R}^{n}}\left(e^{-2 i \pi \gamma \cdot x}-1\right) f(x) d x$.

The purpose of this paper is to establish weighted norm inequalities for $\mathscr{F} f$ with computable constants, for large classes of indices and weights.

This problem is closely related to a recent work of J. J. Benedetto and H. P. Heinig on Fourier inequalities with measure weights $[2,3]$. In these papers, norm inequalities for the Fourier transform were established with few constraints on the weights (e.g. no monotonicity type assumptions) and without rearrangement methods.

As concerns $\mathscr{F} f$, pointwise and norm estimates depending on $A_{p}$ weights were obtained by C. Sadosky and R. L. Wheeden [8]. For a discussion of previous results on weighted Fourier inequalities, we refer to [2, Introduction].

In $[2,3]$, norm inequalities with radial weights were proved for the classical Fourier transform on subspaces of functions with vanishing first moment. Density theorems were established to extend these inequalities to the whole space. Spherical restriction theorems were derived as applications of these results. In this paper, our approach is somewhat different. We establish weighted estimates for $\mathscr{F} f$, for classes of functions for which no moment condition is required. Unless otherwise stated, the weights are not supposed to be radial. We prove $n$-dimensional generalizations of results of [8] concerning pointwise estimates of $\mathscr{F} f(\gamma)$ and negative results for the validity of Fourier inequalities in a specific power weight case. For proving spherical restriction theorems, we use a specific method exploiting the particular properties of the involved measure. This yields a larger range for the indices and a sharper constant. Our study includes a comparison with certain circle restriction properties and a discussion of further types of conditions for the validity of weighted estimates for $\mathscr{F} f$. The results presented in this paper in particular include results of [2 and 3].

Received by the editors July 27, 1990.

1980 Mathematics Subject Classification (1985 Revision). Primary 42B10; Secondary 26D10. 
The general scheme of this paper is the following: $\S 1$ is devoted to norm inequalities for $\mathscr{F} f$. For (possibly nonradial weight functions $u, v, u \in$ $L_{\text {loc }}^{1}\left(\mathbb{R}^{n} \backslash\{0\}\right)$, we first establish inequalities of the form $\|\mathscr{F} f\|_{q, u} \leq C\|f\|_{p, v}$ on the whole of $L_{v}^{p}\left(\mathbb{R}^{n}\right)$. To do this, we find sufficient conditions on $u, v$ in order that the above inequalities hold on the space $L_{c}^{\infty}\left(\mathbb{R}^{n}\right)$ of bounded functions with compact support (Theorem 1.1a). We next observe that, under weaker conditions, $\mathscr{F} f(\gamma)$ exists, as a Lebesgue integral, at every $\gamma \in \mathbb{R}^{n}$ (Lemma 1.1.1). A pointwise estimate also ensures that all the arguments used for proving the norm inequalities in the $L_{c}^{\infty}$ case are still valid for all functions in $L_{v}^{p}$ (Theorem $1.1 \mathrm{a}^{\prime}$, Theorem $1.1 \mathrm{~b}$ ). Using the same method, we obtain analogous conditions for the validity of

$$
\|\mathscr{F} f\|_{q, \mu} \leq C\|f\|_{p, v}
$$

on $L_{v}^{p}$ in the case of a radial measure weight $\mu$ (Theorems $1.2 \mathrm{a}, 1.2 \mathrm{~b}$ ).

In $\S 2$, we derive pointwise and norm approximations for $\mathscr{F} f$, for each $f \in$ $L_{v}^{p}$, in terms of classical Fourier transforms of $L_{c}^{\infty}$ functions (Corollary 2.1). Letting $L_{c, 0}^{\infty} \subset L_{c}^{\infty}$ denote the subspace of functions with integral zero, we show that the previous approximations can be realized by Fourier transforms of functions in $L_{c, 0}^{\infty}$ provided one additional condition is imposed on $v$ (Lemma 2.2, Proposition 2.3, Theorem 2.4).

In $\S 3$, we prove that for $v(x)=|x|^{(n(p-1)}$, no Fourier inequality (in particular no spherical restriction property) can hold for all $f \in L_{c, 0}^{\infty}$ (Theorems 3.1, 3.1' Corollary 3.2).

In $\S 4$, we establish spherical restriction theorems for $\mathscr{F} f$ by using the particular properties of the involved measure and a pointwise estimate of $\mathscr{F} f$ $(\S 4.1)$. In the power weight case, these results are compared for $n=2$ with a circle restriction property of [5] ( $\$ 4.2)$. In $\S 5$, further types of conditions for the boundedness of $\mathscr{F}$ are discussed.

\section{Notation}

For $x \in \mathbb{R}^{n}, \gamma \in \mathbb{R}^{n}$, we use $x \cdot \gamma=\sum_{i=1}^{n} x_{i} \gamma_{i},|x|=\left(\sum_{i=1}^{n} x_{i}^{2}\right)^{1 / 2}$. Also, $B_{a}=\left\{x \in \mathbb{R}^{n}:|x| \leq a\right\}, B_{a}^{c}=\mathbb{R}^{n} \backslash B_{a}, a>0$. The sphere of $\mathbb{R}^{n}$ centered at the origin of radius $\rho>0$ is denoted $S_{n-1}(\rho)$. When $\rho=1$, the abbreviations $S_{n-1}$ or $S$ are used and $\left|S_{n-1}\right|=\int_{S_{n-1}} d \sigma=2 \pi^{n / 2} / \Gamma(n / 2)$.

$L_{c}^{\infty}$ denotes the space of $L^{\infty}$ functions with compact support; $L_{c, 0}^{\infty}=\{f \in$ $\left.L_{c}^{\infty}: \int f d x=0\right\}$. We write $f \in L_{\text {loc }}^{1}(\Omega), \Omega \subset \mathbb{R}^{n}$ if $f \in L^{1}(K)$ for every compact set $K \subset \Omega$.

The space of positive measures on $\mathbb{R}^{n}$ is denoted by $M_{+}\left(\mathbb{R}^{n}\right)$. For $\mu \in$ $M_{+}\left(\mathbb{R}^{n}\right)$, we write $\langle\mu, f\rangle=\int f(t) d \mu(t)$ or simply $\int f d \mu$. For $0<q<$ $\infty, \mu \in M_{+}\left(\mathbb{R}^{n}\right), \quad L_{\mu}^{q}$ is the set of Borel measurable functions defined $\mu$ a.e. on $\mathbb{R}^{n}$ for which $\|f\|_{q, \mu}=\left(\int|f|^{q} d \mu\right)^{1 / q}<\infty$. Similarly, if $v \geq 0$ is a Borel measurable function, not necessarily an element of $L_{\text {loc }}^{1}\left(\mathbb{R}^{n}\right)$, then $L_{v}^{p}=\left\{f:\|f\|_{p, v}=\left(\int|f|^{p} v d t\right)^{1 / p}<\infty\right\}$.

For further remarks on measures, we refer to [2]. Note especially that if $\mu \in M_{+}\left(\mathbb{R}^{n}\right)$ is radial and $\mu(\{0\})=0$, then there exists a unique measure $\nu \in M_{+}(0, \infty)$ such that $\langle\mu, \varphi\rangle=|S| \int_{(0, \infty)} s^{n-1} \varphi(s) d \nu(s)$ for all radial continuous functions $x \rightarrow \varphi(|x|)$ with compact support (cf. [2, Proposition 3.4]). 
When needed, this unique measure $\nu$ is referred to as the measure corresponding to $\mu$. Letting $\sigma_{n-1}$ denote the $(n-1)$-dimensional area measure on $\mathbb{R}^{n}$ and $\mu_{\rho}$ the restriction of $\sigma_{n-1}$ to $S_{n-1}(\rho)$, we note here that the measure $\nu$ corresponding to $\mu_{\rho}$ is $\delta_{\rho}$ (cf. [2, Definition 5.1a]).

For $0<p<\infty$, the conjugate index of $p$ is $p^{\prime}=p /(p-1)$.

The Fourier transform $\hat{f}$ of $f \in L^{1}\left(\mathbb{R}^{n}\right)$ is defined by

$$
\hat{f}(\gamma)=\int_{\mathbb{R}^{n}} e^{-2 i \pi \gamma \cdot x} f(x) d x, \quad \gamma \in \mathbb{R}^{n} .
$$

For suitable functions $f$, we also consider

$$
\mathscr{F} f(\gamma)=\int_{\mathbb{R}^{n}}\left(e^{-2 i \pi \gamma \cdot x}-1\right) f(x) d x
$$

at each $\gamma \in \mathbb{R}^{n}$ where the integral exists.

\section{FourIER INEQUALITIES}

In this section, we prove weighted inequalities for $\mathscr{F} f$ where $\mathscr{F} f$ is defined by $(0.1)$. We first consider the case of weight functions $(\S 1.1)$. We next mention a generalization of a measure weight case considered in [2] (§1.2). In both cases, the estimates are proved to hold on the whole of the $L_{v}^{p}$ space under consideration.

1.1. Fourier inequalities with weight functions. In what follows, the weight functions $u, v$ are not supposed to be radial and $u$ is not required to define a measure on the whole of $\mathbb{R}^{n}$.

In a first stage, we establish inequalities of the form $\|\mathscr{F} f\|_{q, u} \leq C\|f\|_{p, v}$ with $1<p \leq q<\infty$ for all $f \in L_{c}^{\infty}$ (Theorem 1.1a). We next show that, under weaker hypotheses, $\mathscr{F} f(\gamma)$ exists at each $\gamma \in \mathbb{R}^{n}$ and satisfies some pointwise estimates (Lemma 1.1.1, Remark 1.1.2). These imply the validity of the above norm inequalities for all $f \in L_{v}^{p}$ (Theorem 1.1 $\mathrm{a}^{\prime}$ ). An analogous result is mentioned in the case $0<q<p<\infty, p>1$ (Theorem 1.1b).

In the proof of Theorem 1.1a hereafter, we utilize Hardy inequalities with mixed weight functions (see $[1,4])$.

Theorem 1.1a. Let $1<p \leq q<\infty$. Suppose $v \in L_{\text {loc }}^{1}\left(\mathbb{R}^{n}\right), v>0$ a.e. and $v^{1-p^{\prime}} \in L^{1}\left(\mathbb{R}^{n} \backslash\{0\}\right)$. Assume $u$ is nonnegative, $u \in L_{\text {loc }}^{1}\left(\mathbb{R}^{n} \backslash\{0\}\right)$. If

$$
\begin{aligned}
A_{1}= & \sup _{y>0}\left(\left(1 / \pi^{n}\right) \int_{|x|<y}|x|^{q} u(x / \pi) d x\right)^{1 / q} \\
& \cdot\left(\int_{|x|<1 / y}|x|^{p^{\prime}} v(x)^{1-p^{\prime}} d x\right)^{1 / p^{\prime}}<\infty
\end{aligned}
$$

and

$$
\begin{aligned}
A_{2}= & \sup _{y>0}\left(\left(1 / \pi^{n}\right) \int_{|x|>y} u(x / \pi) d x\right)^{1 / q} \\
& \cdot\left(\int_{|x|>1 / y} v(x)^{1-p^{\prime}} d x\right)^{1 / p^{\prime}}<\infty,
\end{aligned}
$$


then there exists $C>0$ such that for all $f \in L_{c}^{\infty}$

$$
\|\mathscr{F} f\|_{q, u} \leq C\|f\|_{p, v}
$$

where $C$ can be chosen as $C=2\left(A_{1}+A_{2}\right)(q)^{1 / q}\left(q^{\prime}\right)^{1 / p^{\prime}}$.

Proof. Suppose $f \in L_{c}^{\infty}$. Then,

$$
\begin{aligned}
\mathscr{F} f(\gamma) & =\int\left(e^{-2 i \pi \gamma \cdot x}-1\right) f(x) d x \\
& =-2 i \int f(t) e^{-i \pi \gamma \cdot t} \sin \pi \gamma \cdot t d t .
\end{aligned}
$$

For $\gamma \neq 0$,

$$
\begin{aligned}
|\mathscr{F} f(\gamma)| & \leq 2 \int|f(t)||\sin \pi \gamma \cdot t| d t \\
& \leq 2 \pi|\gamma| \int_{|t| \leq 1 / \pi|\gamma|}|t||f(t)| d t+2 \int_{|t|>1 / \pi|\gamma|}|f(t)| d t \\
& =2 \pi|\gamma| \cdot F(|\gamma|)+2 G(|\gamma|) .
\end{aligned}
$$

Hence,

$$
\begin{aligned}
\left(\int|\mathscr{F} f(\gamma)|^{q} u(\gamma) d \gamma\right)^{1 / q} \leq & 2 \pi\left(\int|\gamma|^{q} F(|\gamma|)^{q} u(\gamma) d \gamma\right)^{1 / q} \\
& +2\left(\int G(|\gamma|)^{q} u(\gamma) d \gamma\right)^{1 / q} \\
= & 2\left(I_{1}+I_{2}\right) .
\end{aligned}
$$

Explicitly, $I_{1}$ can be written as follows:

$$
I_{1}=\pi\left(\int_{0}^{\infty} F(\rho)^{q} U(\rho) d \rho\right)^{1 / q}
$$

where

$$
\begin{aligned}
& U(\rho)=\rho^{n-1+q} \int_{S} u\left(\rho \gamma^{\prime}\right) d \sigma, \quad \forall^{\prime} \rho \in \mathbb{R} \backslash\{0\}, \\
& F(\rho)=\int_{0}^{1 / \pi \rho} r^{n} \int_{S}|f(r \theta)| d \sigma d r=\int_{\rho}^{\infty} h(s) d s
\end{aligned}
$$

with

$$
h(s)=\pi^{-(n+1)} s^{-(n+2)} \int_{S}|f(\theta / \pi s)| d \sigma, \quad \forall^{\prime} s \in \mathbb{R} \backslash\{0\} .
$$

Therefore, $I_{1}$ is of the form

$$
I_{1}=\pi\left(\int_{0}^{\infty}\left(\int_{\rho}^{\infty} h(s) d s\right)^{q} U(\rho) d \rho\right)^{1 / q} .
$$

By Theorem B of [1], there exists a constant $C_{1}$ such that

$$
I_{1} \leq \pi C_{1}\left(\int_{0}^{\infty} h(t)^{p} V(t) d t\right)^{1 / p}
$$


if $V(t)>0$ a.e. satisfies

$$
A_{1}=\pi \sup _{y>0}\left(\int_{0}^{y / \pi} U(\rho) d \rho\right)^{1 / q}\left(\int_{y / \pi}^{\infty} V(t)^{1-p^{\prime}} d t\right)^{1 / p^{\prime}}<\infty .
$$

Moreover, $A_{1} \leq \pi C_{1} \leq A_{1}(q)^{1 / q}\left(q^{\prime}\right)^{1 / p^{\prime}}$.

The next step, in order to estimate $I_{1}$, is to find $V$ so that

$$
\int_{0}^{\infty} h(t)^{p} V(t) d t \leq \int_{\mathbb{R}^{n}}|f(x)|^{p} v(x) d x .
$$

To that purpose, note that

$$
\int_{0}^{\infty} h(t)^{p} V(t) d t=\pi^{p-1} \int_{0}^{\infty} s^{-p(n+2)}\left(\int_{S}|f(\theta / s)| d \sigma\right)^{p} V(s / \pi) d s .
$$

Applying Hölder's inequality to the inside integral over $S$, we see that the right side of the above equality is dominated by

$$
\begin{array}{r}
\pi^{p-1} \int_{0}^{\infty} s^{-p(n+2)}\left(\int_{S}|f(\theta / s)|^{p} v(\theta / s) d \sigma\right) \\
\times\left(\int_{S} v(\theta / s)^{-p^{\prime} / p} d \sigma\right)^{p / p^{\prime}} V(s / \pi) d s .
\end{array}
$$

Since, on the other hand,

$$
\int_{\mathbb{R}^{n}}|f(x)|^{p} v(x) d x=\int_{0}^{\infty} s^{-(n+1)}\left(\int_{S}|f(\theta / s)|^{p} v(\theta / s) d \sigma\right) d s,
$$

we see that (1.4) will be satisfied if

$$
V(s / \pi)=\pi^{1-p} s^{p(n+2)-(n+1)}\left(\int_{S} v(\theta / s)^{-P^{\prime} / p} d \sigma\right)^{-p / p^{\prime}} .
$$

With this choice of $V$, we thus obtain $I_{1} \leq \pi C_{1}\|f\|_{p, v}$. Moreover, as simple calculations show,

$$
\begin{aligned}
\int_{y / \pi}^{\infty} V(t)^{1-p^{\prime}} d t & =\int_{0}^{1 / y} t^{p^{\prime}+n-1}\left(\int_{S} v(\theta t)^{1-p^{\prime}} d \sigma\right) d t \\
& =\int_{|x|<1 / y}|x|^{p^{\prime}} v(x)^{1 / p^{\prime}} d x
\end{aligned}
$$

and

$$
\int_{0}^{y / \pi} U(\rho) d \rho=\pi^{-(q+n)} \int_{|x|<y}|x|^{q} u(x / \pi) d x .
$$

Therefore, the constant $A_{1}$ introduced above is equal to the constant defined in (1.1).

In order to estimate $I_{2}$, we use a similar method. In this case,

$$
I_{2}=\left(\int_{0}^{\infty} G(\rho)^{q} \rho^{-q} U(\rho) d \rho\right)^{1 / q},
$$

where $G(\rho)=\int_{0}^{\rho} \pi s h(s) d s$ with $U(\rho), h(s)$ defined as before. Theorem A of [1] then yields

$$
I_{2} \leq C_{2}\left(\int_{0}^{\infty}(\pi s)^{p} h(s)^{p} W(s) d s\right)^{1 / p}
$$


if $W>0$ a.e. is such that

$$
A_{2}=\sup _{y>0}\left(\int_{y / \pi}^{\infty} \rho^{-q} U(\rho) d \rho\right)^{1 / q}\left(\int_{0}^{y / \pi} W(t)^{1-p^{\prime}} d t\right)^{1 / p^{\prime}}<\infty .
$$

Moreover, $A_{2} \leq C_{2} \leq A_{2}(q)^{1 / q}\left(q^{\prime}\right)^{1 / p^{\prime}}$.

If we choose $W(s)=(\pi s)^{-p} V(s)$ where $V$ is the auxiliary weight introduced for estimating $I_{1}$, inequality (1.4) immediately implies $I_{2} \leq C_{2}\|f\|_{p, v}$. Moreover, it is readily seen that $A_{2}$ is equal to the constant defined in (1.2).

From the estimates of $I_{1}, I_{2}$, it now follows that (1.3) is satisfied for all $f \in L_{c}^{\infty}$ with $C=\pi C_{1}+C_{2}$. This ends the proof.

In order to establish Fourier inequalities for all $f \in L_{v}^{p}$, we now prove the following $n$-dimensional generalization of a fact already pointed out in [8] in a one-dimensional case.

Lemma 1.1.1. Suppose $1<p<\infty, v \in L_{\text {loc }}^{1}\left(\mathbb{R}^{n}\right), v>0$ a.e. If $|t|^{p^{\prime}} v^{1-p^{\prime}} \in$ $L_{\text {loc }}^{1}\left(\mathbb{R}^{n}\right)$ and $v^{1-p^{\prime}} \in L^{1}\left(B_{a}^{c}\right)$ for some $a>0$, then, for all $f \in L_{v}^{p}\left(\mathbb{R}^{n}\right), \mathscr{F} f(\gamma)$ exists, as a Lebesgue integral, at each $\gamma \in \mathbb{R}^{n}$. Moreover

$$
|\mathscr{F} f(\gamma)| \leq K(|\gamma|, v) \cdot\|f\|_{p, v}
$$

for all $\gamma \in \mathbb{R}^{n}$, where

$$
\begin{gathered}
K(\rho, v)=2 \inf _{a>0} K_{a}(\rho, v) \\
K_{a}(\rho, v)=\left\{(\pi \rho)^{p^{\prime}} \int_{|t| \leq a}|t|^{p^{\prime}} v^{1-p^{\prime}} d t+\int_{|t|>a} v^{1-p^{\prime}} d t\right\}^{1 / p^{\prime}}
\end{gathered}
$$

for each $\rho>0, a>0$.

Proof. Let $f \in L_{v}^{p}$ and $\gamma \neq 0$. For every $a>0$,

$$
|f(t) \sin \pi \gamma \cdot t| \leq \begin{cases}\pi|\gamma||t||f(t)| & \text { a.e. for }|t| \leq a \\ |f(t)| & \text { a.e. for }|t|>a\end{cases}
$$

From the following Hölder inequalities

$$
\begin{gathered}
\pi|\gamma| \int_{|t| \leq a}|t||f(t)| d t \leq \pi|\gamma|\left(\int_{|t| \leq a}|f|^{p} v d t\right)^{1 / p}\left(\int_{|t| \leq a}|t|^{p^{\prime}} v^{1-p^{\prime}} d t\right)^{1 / p^{\prime}}, \\
\int_{|t|>a}|f(t)| d t \leq\left(\int_{|t|>a}|f|^{p} v d t\right)^{1 / p}\left(\int_{|t|>a} v^{1-p^{\prime}} d t\right)^{1 / p^{\prime}},
\end{gathered}
$$

it then follows that

$$
|\mathscr{F} f(\gamma)| \leq 2 \int|f||\sin \pi \gamma \cdot t| d t \leq K(|\gamma|, v) \cdot\|f\|_{p, v}
$$

where $K(\rho, v)$ is defined by (1.6) for $\rho>0$.

Note also that the above majorizations and Lebesgue's theorem imply that $\mathscr{F} f$ is a continuous function on $\mathbb{R}^{n}$. 
Remark 1.1.2. The following calculation shows that for each $\rho>0$, the infimum of $K_{a}(\rho, v)$ over $a>0$ is attained for $a=a_{0}=1 /(\pi \rho)$. Indeed for $a>a_{0}$, and each fixed $\rho>0$,

$$
\begin{aligned}
K_{a}(\rho, v)^{p^{\prime}} & =K_{a_{0}}(\rho, v)^{p^{\prime}}+\int_{a_{0}<|t|<a}\left((\pi \rho|t|)^{p^{\prime}}-1\right) v^{1-p^{\prime}} d t \\
& \geq K_{a_{0}}(\rho, v)^{p^{\prime}} .
\end{aligned}
$$

A similar conclusion holds for $a<a_{0}$, which yields $\inf _{0<a<\infty} K_{a}(\rho, v)=$ $K_{1 /(\pi \rho)}(\rho, v)$.

In view of Lemma 1.1.1, all the majorizations and arguments developed in the proof of Theorem 1.1 a also apply to all functions of $L_{v}^{p}$. We can thus state:

Theorem 1.1a'. Suppose $p, q, u, v$ as in Theorem 1.1a. Assume $A_{1}<\infty$, $A_{2}<\infty$. Then,

$$
\|\mathscr{F} f\|_{q, u} \leq C\|f\|_{p, v}
$$

holds for all $f \in L_{v}^{p}$, where $\mathscr{F} f(\gamma)$ exists, as a Lebesgue integral, for each $\gamma \in \mathbb{R}^{n}$. The constant $C$ is the same as in Theorem 1.1a.

As this was pointed out to us by H. P. Heining, the method we used for proving $\left(1.3^{\prime}\right)$ for $1<p \leq q<\infty$ also applies to the case $0<q<p<$ $\infty, p>1$. In this case however, the conditions for the validity of Hardy type inequalities are different (see $[3,6,9])$. The corresponding result is the following.

Theorem 1.1b. Suppose $0<q<p<\infty, p>1,1 / r=1 / q-1 / p$. Assume $v \in L_{\text {loc }}^{1}\left(\mathbb{R}^{n}\right), v>0$ a.e., $v^{1-p^{\prime}} \in L^{1}\left(\mathbb{R}^{n} \backslash\{0\}\right)$, and $u$ is nonnegative, $u \in$ $L_{\text {loc }}^{1}\left(\mathbb{R}^{n} \backslash\{0\}\right)$. If

$$
\begin{aligned}
B_{1}=\int_{\mathbb{R}^{n}} & \left(\int_{|x| \leq 1 /(\pi|y|)} u(x)|x|^{q} d x\right)^{r / q} \\
& \cdot\left(\int_{|x| \leq|y|}|x|^{p^{\prime}} v^{1-p^{\prime}} d x\right)^{r / q^{\prime}}|y|^{p^{\prime}} v(y)^{1-p^{\prime}} d y<\infty
\end{aligned}
$$

and

$$
\begin{aligned}
B_{2}=\int_{\mathbb{R}^{n}} & \left(\int_{|x| \geq 1 /(\pi|y|)} u(x) d x\right)^{r / q} \\
& \cdot\left(\int_{|x| \geq|y|} v(x)^{1-p^{\prime}} d x\right)^{r / q^{\prime}} v(y)^{1-p^{\prime}} d y<\infty,
\end{aligned}
$$

then, there exists a constant $C>0$ such that $\|\mathscr{F} f\|_{q, u} \leq C\|f\|_{p, v}$ for all $f \in L_{v}^{p}$.

1.2. Fourier inequalities with measure weights. We now mention the analogue of Theorem $1.1 \mathrm{a}^{\prime}$ when $u$ is replaced by a radial measure weight $\mu$ on $\mathbb{R}^{n}$. The result includes Theorem 4.1 of [2]. The proof utilizes two specific arguments used in [2], namely, the representation of certain radial measures [2, Proposition 3.4] and Hardy inequalities with measure weights [10, 2, Theorem 1.1]. In addition, nonradial techniques similar to those used in $\S 1.1$ are required. 
Theorem 1.2a. Let $1<p \leq q<\infty$. Suppose $v \in L_{\mathrm{loc}}^{1}\left(\mathbb{R}^{n}\right), v>0$ a.e., $v^{1-p^{\prime}} \in$ $L_{\text {loc }}^{1}\left(\mathbb{R}^{n} \backslash\{0\}\right)$. Suppose $\mu \in M_{+}\left(\mathbb{R}^{n}\right)$ is radial, $\mu(\{0\})=0$. Let $\nu \in M_{+}(0, \infty)$ denote the measure corresponding to $\mu$. If

$$
A_{1}^{*}=\sup _{y>0}\left(\int_{(0, y)} s^{n-1+q} d \nu(s / \pi)\right)^{1 / q}\left(\int_{|t|<1 / y}|t|^{p^{\prime}} v^{1-p^{\prime}} d t\right)^{1 / p^{\prime}}<\infty
$$

and

$$
A_{2}^{*}=\sup _{y>0}\left(\int_{(y, \infty)} s^{n-1} d \nu(s / \pi)\right)^{1 / q}\left(\int_{|t|>1 / y} v^{1-p^{\prime}} d t\right)^{1 / p^{\prime}}<\infty
$$

then there exists $C^{*}>0$ such that

$$
\|\mathscr{F} f\|_{q, \mu} \leq C^{*}\|f\|_{p, v}
$$

for all $f \in L_{v}^{p}$ where $C^{*}$ can be chosen as

$$
C^{*}=2 \pi^{-(n-1) / q}\left|S_{n-1}\right|^{1 / q}(p)^{1 / q}\left(p^{\prime}\right)^{1 / p^{\prime}}\left(A_{1}^{*}+A_{2}^{*}\right) .
$$

A similar result generalizing [3, Theorem 4.1] holds in case $0<q<p<\infty$, $p>1$ :

Theorem 1.2b. Let $0<q<p<\infty, p>1,1 / r=1 / q-1 / p$. Suppose $v$ and $\mu$ are as in Theorem 1.2a. If

$$
\begin{aligned}
B_{1}^{*}=\int_{\mathbb{R}^{n}} & \left(\int_{(0,1 /|y|)} \rho^{n-1+q} d \nu(\rho / \pi)\right)^{r / q} \\
& \cdot\left(\int_{|x| \leq|y|}|x|^{p^{\prime}} v^{1-p^{\prime}} d x\right)^{r / q^{\prime}}|y|^{p^{\prime}} v(y)^{1-p^{\prime}} d y<\infty
\end{aligned}
$$

and

$$
\begin{aligned}
B_{2}^{*}= & \int_{\mathbb{R}^{n}}\left(\int_{(1 /|y|, \infty)} \rho^{n-1} d \nu(\rho / \pi)\right)^{r / q} \\
& \cdot\left(\int_{|x| \geq|y|} v^{1-p^{\prime}} d x\right)^{r / q^{\prime}} v(y)^{1-p^{\prime}} d y<\infty,
\end{aligned}
$$

then there exists $C^{*}$ such that $\|\mathscr{F} f\|_{q, \mu} \leq C^{*}\|f\|_{p, v}$ for all $f \in L_{v}^{p}$.

\section{Approximations of $\mathscr{F} f$}

Since $L_{c}^{\infty}$ is dense in $L_{v}^{p}$ whenever $v \in L_{\text {loc }}^{1}$, we can deduce the following pointwise and norm approximations of $\mathscr{F} f$ from Lemma 1.1.1 and Theorem $1.1 \mathrm{a}^{\prime}$ (respectively Theorem 1.2a).

Corollary 2.1. Suppose the assumptions of Theorem 1.1 a' (respectively Theorem 1.2a) are satisfied. Then, for each fixed $f \in L_{v}^{p}\left(\mathbb{R}^{n}\right)$,

(i) $\mathscr{F} f(\gamma)=\lim _{m}\left(\hat{f}_{m}(\gamma)-\hat{f}_{m}(0)\right)$ for every $\gamma \in \mathbb{R}^{n}$;

(ii) $\mathscr{F} f=\lim _{m}\left(\hat{f}_{m}-\hat{f}_{m}(0)\right)$ in the $L_{u}^{q}$ norm (respectively the $L_{\mu}^{q}$ norm) whenever $f_{m} \in L_{c}^{\infty}$ is an approximating sequence of $f$ in the $L_{v}^{p}$ norm.

Similar properties hold in the case $0<q<p<\infty, p>1$ if the assumptions of Theorem $1.1 \mathrm{~b}$ (respectively $1.2 \mathrm{~b}$ ) are satisfied. 
In order to approximate $\mathscr{F} f$ by Fourier transforms of functions with vanishing first moment, we now mention a simple proof of a density lemma regarding the subspace $L_{c, 0}^{\infty}\left(\mathbb{R}^{n}\right)$ of $L_{v}^{p}\left(\mathbb{R}^{n}\right)$, where $v \in L_{\text {loc }}^{1}\left(\mathbb{R}^{n}\right), v>0$ a.e. Analogous theorems were previously established in [2] with a different method of proof, for the moment subspace

$$
M_{0}(n) \cap L_{v}^{p}\left(\mathbb{R}^{n}\right), \quad M_{0}(n)=\left\{f \in L^{1}\left(\mathbb{R}^{n}\right): \int f d x=0\right\},
$$

under the stronger hypothesis, $v \in L^{r}\left(\mathbb{R}^{n}\right)$ form some $r>1$ (see [2, Theorems $2.2,4.2])$.

Note. After we wrote the following lemma, John J. Benedetto informed us that J. Garcia-Cuerva, C. Heil, S. Saeki independently observed that the $L_{\text {loc }}^{r}$ condition, $r>1$, in [2] could be replaced by the condition $v \in L_{\text {loc }}^{1}$. S. Saeki also showed him a proof of the density result which was very much like ours.

Lemma 2.2. Suppose $1<p<\infty, v \in L_{\text {loc }}^{1}\left(\mathbb{R}^{n}\right), v>0$ a.e.

(i) If $h \in\left(L_{v}^{p}\left(\mathbb{R}^{n}\right)\right)^{\prime}$ annihilates $L_{c, 0}^{\infty}\left(\mathbb{R}^{n}\right)$, then $h$ is a constant function.

(ii) $\overline{L_{c, 0}^{\infty}\left(\mathbb{R}^{n}\right)}=L_{v}^{p}\left(\mathbb{R}^{n}\right)$ or $L_{v}^{p}\left(\mathbb{R}^{n}\right) \subset L^{1}\left(\mathbb{R}^{n}\right)$.

(iii) If $v^{1-p^{\prime}} \notin L^{1}\left(\mathbb{R}^{n}\right)$, then $\overline{L_{c, 0}^{\infty}\left(\mathbb{R}^{n}\right)}=L_{v}^{p}\left(\mathbb{R}^{n}\right)$.

Proof. (i) Suppose $h \in\left(L_{v}^{p}\left(\mathbb{R}^{n}\right)\right)^{\prime}$ i.e., $h \in L_{v^{1-p^{\prime}}}^{p^{\prime}}\left(\mathbb{R}^{n}\right)$. Since $v \in L_{\text {loc }}^{1}\left(\mathbb{R}^{n}\right)$, $v>0$ a.e., it follows from Hölder's inequality that $h \in L_{\text {loc }}^{1}\left(\mathbb{R}^{n}\right)$.

Let $\xi(x)=\left(n /\left|S_{n-1}\right|\right) \chi_{|x| \leq 1}(x)$ and $\xi_{m}(x)=\left(1 / m^{n}\right) \cdot \xi(x / m), m>0$. Then, $\int_{\mathbb{R}^{n}} \xi_{m}(t-\tau) d t=1$ for every $\tau \in \mathbb{R}^{n}$ and every $m>0$.

Suppose $f \in L_{c}^{\infty}$ such that $a_{f}=\int f d x \neq 0$. Define $f_{m, \tau}=f-\xi_{m}(\cdot-\tau) \cdot a_{f}$. Then, $f_{m, \tau} \in L_{c, 0}^{\infty}$ for every $m>0$ and $\tau \in \mathbb{R}^{n}$ and if $h$ annihilates $L_{c, 0}^{\infty}$, one has $\left\langle h, f_{m, \tau}\right\rangle=\langle h, f\rangle-a_{f}\left\langle h, \xi_{m}(\cdot-\tau)\right\rangle=0$ for all $m>0$ and $\tau \in \mathbb{R}^{n}$. Thus,

$$
\langle h, f\rangle=\left(n /\left|S_{n-1}\right|\right) a_{f} \cdot \frac{1}{m^{n}} \int_{|t-\tau| \leq m} h(t) d t
$$

for all $m>0, \tau \in \mathbb{R}^{n}$. Since $h \in L_{\text {loc }}^{1}\left(\mathbb{R}^{n}\right)$, the right-hand side of this equality tends to $a_{f} \cdot h(\tau)$, at each Lebesgue point $\tau$ of $h$ when $m \rightarrow 0+$. Hence, for almost every fixed $\tau \in \mathbb{R}^{n}, \int(h(t)-h(\tau)) f(t) d t=0$ for all $f \in L_{c}^{\infty}$. This yields $h(t)=h(\tau)$ a.e. and assertion (i) follows.

(ii) and (iii) follow from (i) in the same way as in [2, proof of Theorem 2.2]: if $h(t)=k=0$, then $\overline{L_{c, 0}^{\infty}\left(\mathbb{R}^{n}\right)}=L_{v}^{p}\left(\mathbb{R}^{n}\right)$. If $h \in L_{v^{1-p^{\prime}}}^{p^{\prime}}\left(\mathbb{R}^{n}\right), h(t)=k \neq 0$, then $\int|h|^{p^{\prime}} v^{1-p^{\prime}} d t=|k|^{p^{\prime}} \int v^{1-p^{\prime}} d t<\infty$ i.e., $v^{1-p^{\prime}} \in L^{1}\left(\mathbb{R}^{n}\right)$. Consequently, $\int|f| d t<\infty$ for $f \in L_{v}^{p}$. This ends the proof.

In connection with assertion (iii) of the above lemma, we give now a criterion for the validity of condition $v^{1-p^{\prime}} \notin L^{1}\left(\mathbb{R}^{n}\right)$. This is an $n$-dimensional generalization, with a different proof, of [2, Proposition 2.6] (see also [7, Theorem 6.19] for related one-dimensional results).

Proposition 2.3. Suppose $1<p<\infty, v \in L_{\mathrm{loc}}^{1}\left(\mathbb{R}^{n}\right), v>0$ a.e. If

$$
\varliminf_{m \rightarrow \infty} \frac{1}{m^{n p}}\left(\int_{|t| \leq m} v(t) d t\right)=0
$$

then $v^{1-p^{\prime}} \notin L^{1}\left(\mathbb{R}^{n}\right)$. 
Proof. Suppose $v^{1-p^{\prime}} \in L^{1}\left(\mathbb{R}^{n}\right)$. Then, for every $m \geq 1$,

$$
\frac{\left|S_{n-1}\right|}{n}=\frac{1}{m^{n}} \int_{|x| \leq m} d x \leq \frac{1}{m^{n}}\left(\int_{|x| \leq m} v(t) d t\right)^{1 / p} \cdot\left\|v^{1-p^{\prime}}\right\|_{1}^{1 / p^{\prime}} .
$$

Since the left side is a strictly positive constant, the result follows.

Combining the above density lemma with Lemma 1.1 .1 and Theorem $1.1 \mathrm{a}^{\prime}$ (respectively Theorem $1.2 \mathrm{a}$ ) we now obtain

Theorem 2.4. Suppose the assumptions of Theorem 1.1 $\mathrm{a}^{\prime}$ (respectively Theorem $1.2 \mathrm{a})$ are satisfied. Assume moreover that $v^{1-p^{\prime}}$ is not locally integrable in a neighborhood of the origin. Then, for each fixed $f \in L_{v}^{p}\left(\mathbb{R}^{n}\right)$ and any sequence $f_{m} \in L_{c, 0}^{\infty}\left(\mathbb{R}^{n}\right)$ approximating $f$ in the $L_{v}^{p}$ norm,

(i) $\mathscr{F} f(\gamma)=\lim _{m} \hat{f}_{m}(\gamma)$ at each $\gamma \in \mathbb{R}^{n}$;

(ii) $\mathscr{F} f=\lim _{m} \hat{f}_{m}$ in the $L_{u}^{q}$ norm (respectively, the $L_{\mu}^{q}$ norm).

Similar results hold in the case $0<q<p<\infty, p>1$ if the assumptions of Theorem $1.1 \mathrm{~b}$ (respectively $1.2 \mathrm{~b}$ ) are satisfied together with the additional condition $v \notin L^{1}\left(\mathbb{R}^{n}\right)$.

Note. Assertion (ii) above in particular means that $\mathscr{F}: L_{v}^{p} \rightarrow L_{u}^{q}$ (respectively $L_{\mu}^{q}$ ) is the bounded extension of the Fourier operator $f \rightarrow \hat{f}$ initially defined on $L_{c, 0}^{\infty}$.

After we wrote the results of this section, J. Benedetto informed us that J. Lakey independently observed that $\mathscr{F}$ was the bounded extension of $f \rightarrow$ $\hat{f}: M_{0}(n) \cap L_{v}^{p} \rightarrow L_{\mu}^{q}$ in the case of the $L_{v}^{p}$ spaces considered in [2]. J. Benedetto and $J$. Lakey have also obtained results on the extension problem in terms of Wiener amalgam spaces.

\section{AN $n$-DIMENSIONAL COUNTEREXAMPLE}

In Theorem 3.1 below, we show that the inequality $\|\hat{f}\|_{q, \mu} \leq C\|f\|_{p, v}$ fails when $v(x)=|x|^{n(p-1)}$. To this end, we construct a counterexample which generalizes a one-dimensional counterexample of [8] to the case of measure weights in $\mathbb{R}^{n}, n \geq 2$.

Theorem 3.1. Let $n>1,0<q<\infty, 1<p<\infty$. Suppose $\mu \in M_{+}\left(\mathbb{R}^{n}\right), \mu$ not identically zero on $\mathbb{R}^{n}, \mu(\{0\})=0$. Then, there does not exist a constant $C>0$ such that

$$
\left(\int_{\mathbb{R}^{n}}|\hat{f}(\gamma)|^{q} d \mu(\gamma)\right)^{1 / q} \leq C\left(\int_{\mathbb{R}^{n}}|f(x)|^{p}|x|^{n(p-1)} d x\right)^{1 / p}
$$

holds for all $f \in L_{c, 0}^{\infty}\left(\mathbb{R}^{n}\right)$.

Proof. For $N>1$, define

$$
F_{N}(x)=\frac{1}{|x|^{n}}\left\{\chi_{(1 / N, 1)}(|x|)-\chi_{(1, N)}(|x|)\right\} .
$$

As it can easily be seen, $F_{N} \in L_{c, 0}^{\infty}$ and

$$
\left(\int_{\mathbb{R}^{n}}\left|F_{N}(x)\right|^{p}|x|^{n(p-1)} d x\right)^{1 / p}=\left(2\left|S_{n-1}\right| \ln N\right)^{1 / p} .
$$


In order to minorize $\left|\widehat{F}_{N}(\gamma)\right|$, note that

$$
\begin{aligned}
\left|\widehat{F}_{N}(\gamma)\right| & =\left|\int_{1 / N<|t|<1} \frac{e^{-2 i \pi \gamma \cdot t}}{|t|^{n}} d t-\int_{1<|t|<N} \frac{e^{-2 i \pi \gamma \cdot t}}{|t|^{n}} d t\right| \\
& =\left|\int_{1 / N<|t|<1} \frac{d t}{|t|^{n}}+\int_{1 / N<|t|<1} \frac{e^{-2 i \pi \gamma \cdot t}-1}{|t|^{n}} d t-\int_{1<|t|<N} \frac{e^{-2 i \pi \gamma \cdot t}}{|t|^{n}} d t\right| \\
& =|| S_{n-1}\left|\ln N+A_{N}(\gamma)-B_{N}(\gamma)\right| \\
& \geq\left|S_{n-1}\right| \ln N-\left|A_{N}(\gamma)\right|-\left|B_{N}(\gamma)\right|,
\end{aligned}
$$

where

$$
\left|A_{N}(\gamma)\right| \leq 2 \pi|\gamma| \int_{1 / N<|t|<1} \frac{d t}{|t|^{n-1}} \leq 2 \pi|\gamma| \cdot\left|S_{n-1}\right| .
$$

Noting $\gamma=|\gamma| \Theta, \quad \Theta \in S_{n-1}$, we can write

$$
B_{N}(\gamma)=B_{N}(|\gamma| \theta)=\int_{1}^{N} \int_{S_{n-1}} e^{-2 i \pi \rho|\gamma| \omega \cdot \theta} d \sigma_{n-1}(\omega) \frac{d \rho}{\rho},
$$

where the inner integral is equal to

$$
\begin{aligned}
& \left|S_{n-2}\right| \int_{0}^{\pi} e^{-2 i \pi \rho|\gamma| \cos \alpha}(\sin \alpha)^{n-2} d \alpha \\
& \quad=\left|S_{n-2}\right| \int_{-1}^{+1} e^{-2 i \pi \rho|\gamma| t}\left(1-t^{2}\right)^{(n-3) / 2} d t \\
& \quad=2\left|S_{n-2}\right| \int_{0}^{1} \cos (2 \pi \rho|\gamma| t) \cdot\left(1-t^{2}\right)^{(n-3) / 2} d t .
\end{aligned}
$$

In the case $n=2$, this equality is true with $\left|S_{0}\right|=2$.

Let $\psi_{n}(t)=\int_{0}^{t}\left(1-u^{2}\right)^{(n-3) / 2} d u$. This function is continuous and increasing on $[0,1]$ for all $n \geq 2$. Moreover, $2\left|S_{n-2}\right| \psi_{n}(1)=\left|S_{n-1}\right|, n \geq 2$. By integrating by parts, we see that the integral in the right side of (3.4) is equal to

$$
\cos (2 \pi \rho|\gamma|) \cdot \psi_{n}(1)+2 \pi \rho|\gamma| \int_{0}^{1} \sin (2 \pi \rho|\gamma| t) \psi_{n}(t) d t .
$$

Consequently, since

$$
\begin{aligned}
2 \pi|\gamma| & \cdot\left|\int_{1}^{N} \int_{0}^{1} \sin (2 \pi \rho|\gamma| t) \psi_{n}(t) d t d \rho\right| \\
& \leq 2 \int_{0}^{1}\left(\psi_{n}(t) / t\right) d t<\infty
\end{aligned}
$$

and

$$
\begin{aligned}
\left|\int_{1}^{N} \frac{\cos (2 \pi \rho|\gamma|)}{\rho} d \rho\right| & =\mid \frac{\sin (2 \pi|\gamma| N)}{2 \pi|\gamma| N}-\frac{\sin (2 \pi|\gamma|)}{2 \pi|\gamma|} \\
& +\frac{1}{2 \pi|\gamma|} \int_{1}^{N} \frac{\sin (2 \pi \rho|\gamma|)}{\rho^{2}} d \rho \mid \\
& \leq \frac{1}{2 \pi|\gamma|}\left(\frac{1}{N}+1+1-\frac{1}{N}\right)=\frac{1}{\pi|\gamma|}
\end{aligned}
$$


we obtain $\left|B_{N}(\gamma)\right| \leq\left|S_{n-1}\right|\left(1 / \pi|\gamma|+k_{n}\right)$, where $k_{n}$ is a constant depending only on $n$.

From (3.3) and the above estimate, it follows that

$$
\left|\widehat{F}_{N}(\gamma)\right| \geq\left|S_{n-1}\right|\left(\ln N-2 \pi|\gamma|-\frac{1}{\pi|\gamma|}-k_{n}\right) .
$$

Since $\mu(\{0\})=0$ and $\mu$ is not identically zero on $\mathbb{R}^{n}$, there exists $a, b$, $0<a<b<\infty$ such that $\int_{a<|\gamma|<b} d \mu(\gamma) \neq 0$.

Choosing $N_{0}=N_{0}(a, b)$ such that $\frac{1}{2} \ln N_{0}>2 \pi b+1 / \pi a+k_{n}$, we see that for $a<|\gamma|<b$, the inequality

$$
\left|\widehat{F}_{N}(\gamma)\right| \geq\left|S_{n-1}\right|\left(\ln N-2 \pi|\gamma|-\frac{1}{\pi|\gamma|}-k_{n}\right)>\left|S_{n-1}\right| \frac{\ln N}{2}
$$

holds for all $N \geq N_{0}$. Since $\mu \in M_{+}\left(\mathbb{R}^{n}\right)$, this yields

$$
\left(\int_{\mathbb{R}^{n}}\left|\widehat{F}_{N}(\gamma)\right|^{q} d \mu(\gamma)\right)^{1 / q} \geq\left(\frac{1}{2}\left|S_{n-1}\right| \ln N\right)\left(\int_{a<|\gamma|<b} d \mu(\gamma)\right)^{1 / q}
$$

for all $N \geq N_{0}$. If the Fourier inequality (3.1) were true, it would follow from (3.2) that

$$
0<\left(\int_{a<|\gamma|<b} d \mu(\gamma)\right)^{1 / q}(\ln N)^{1 / p^{\prime}} \leq \text { const . }
$$

which is impossible when $p>1$. This ends the proof.

In the important case where $\mu$ is the restriction of $\sigma_{n-1}$ to the unit sphere $S_{n-1}$ of $\mathbb{R}^{n}$, Theorem 3.1 yields:

Corollary 3.2. Let $n>1,0<q<\infty, 1<p<\infty, v(x)=|x|^{n(p-1)}$. Then, no spherical restriction property $\|\hat{f}\|_{L^{q}\left(S_{n-1}\right)} \leq C\|f\|_{p, v}$ with $C$ constant, can hold for all $f \in L_{c, 0}^{\infty}$.

Note also the following variant of Theorem 3.1 where the measure $\mu$ is replaced by a nonnegative function $u \in L_{\text {loc }}^{1}\left(\mathbb{R}^{n} \backslash\{0\}\right)$.

Theorem 3.1'. Let $n>1,0<q<\infty, 1<p<\infty$. Suppose $u \in L_{\text {loc }}^{1}\left(\mathbb{R}^{n} \backslash\{0\}\right)$, $u$ is nonnegative and not almost everywhere equal to zero. Assume $v(x)=$ $|x|^{n(p-1)}$. Then, there does not exist $C>0$ such that $\|\hat{f}\|_{q, u} \leq C\|f\|_{p, v}$ holds for all $f \in L_{c, 0}^{\infty}$.

\section{WEIGHTED RESTRICTION PROPERTIES FOR $\mathscr{F} f$}

4.1. Denote by $S_{n-1}(\rho)$ the sphere of radius $\rho>0$ centered at the origin in $\mathbb{R}^{n}$ and by $\mu_{\rho}$ the restriction of $\sigma_{n-1}$ to $S_{n-1}(\rho)$. Note also $S=S_{n-1}(1)$.

In what follows, we establish estimates of the form

$$
\left(\int_{S_{n-1}(\rho)}|\mathscr{F} f(\gamma)|^{q} d \sigma_{n-1}\right)^{1 / q} \leq L(\rho, v) \cdot\|f\|_{p, v}
$$

where $v$ is a possibly nonradial weight, $1<p<\infty, 0<q<\infty$. Our method of proof differs from that of [2]. In the latter paper, spherical restriction 
properties for $\hat{f}$ are obtained by applying some previously established Fourier inequalities to the particular measure $\mu_{\rho}$. Here, we derive inequalities of type (4.1) by integrating the pointwise estimate of $\mathscr{F} f(\gamma)$ furnished by Lemma 1.1.1. This method leads to a larger range for $p, q$ and a sharper constant $L(\rho, v)$.

Theorem 4.1. Let $1<p<\infty, 0<q<\infty$, and $v \in L_{\mathrm{loc}}^{1}\left(\mathbb{R}^{n}\right), v>0$ a.e. Suppose $|t|^{p^{\prime}} v^{1-p^{\prime}} \in L_{\mathrm{loc}}^{1}\left(\mathbb{R}^{n}\right)$ and $v^{1-p^{\prime}} \in L^{1}\left(B_{a}^{c}\right)$ for some $a>0$. Then,

(i) for each $\rho>0$,

$$
\left(\int_{S_{n-1}(\rho)}|\mathscr{F} f(\gamma)|^{q} d \sigma_{n-1}\right)^{1 / q} \leq|S|^{1 / q} \rho^{(n-1) / q} K(\rho, v)\|f\|_{p, v}
$$

for all $f \in L_{v}^{p}$, where $K(\rho, v)$ is defined by (1.6).

(ii) for each $\rho>0$ and each fixed $f \in L_{v}^{p}, \mathscr{F} f=\lim _{m}\left(\hat{f}_{m}-\hat{f}_{m}(0)\right)$ in the pointwise sense everywhere and in the $L^{q}\left(S_{n-1}(\rho)\right)$ norm, whenever $f_{m} \in L_{c}^{\infty}$ is an approximating sequence of $f$ in the $L_{v}^{p}$ norm.

Remark. In the particular case of radial weights, the constant in (i) is sharper than the analogous one in [2, Theorem 5.3]. Theorem 4.1 also includes [ 3 , Theorem 4.2].

Proof. (i) By Lemma 1.1.1, $|\mathscr{F} f(\gamma)| \leq\|f\|_{p, v} \cdot 2 K_{a}(|\gamma|, v)$ for all $\gamma \in \mathbb{R}^{n}$ and every $a>0$, where

$$
K_{a}(|\gamma|, v)=\left((\pi|\gamma|)^{p^{\prime}} \int_{|t| \leq a}|t|^{p^{\prime}} v^{1-p^{\prime}} d t+\int_{|t|>a} v^{1-p^{\prime}} d t\right)^{1 / p^{\prime}} .
$$

Therefore, for each fixed $\rho>0$, we have for any $q>0$ and any $a>0$,

$$
\begin{aligned}
& \left(\int_{S_{n-1}(\rho)}|\mathscr{F} f(\gamma)|^{q} d \sigma\right)^{1 / q} \leq 2\|f\|_{p, v}\left(\int_{S_{n-1}(\rho)} K_{a}(\rho, v)^{q} d \sigma\right)^{1 / q} \\
& \quad=2\|f\|_{p, v}\left(\left\langle|S| \rho^{n-1} \delta(|\gamma|-\rho), K_{a}(|\gamma|, v)^{q}\right\rangle\right)^{1 / q} \\
& \quad=2\|f\|_{p, v}|S|^{1 / q} \rho^{(n-1) / q} K_{a}(\rho, v)
\end{aligned}
$$

which yields (4.2).

(ii) follows from (i) and Lemma 1.1.1.

In view of the density Lemma 2.2 , the preceding approximations can be realized by Fourier transforms of functions with integral zero provided an additional hypothesis on the weight $v$ is satisfied. We thus obtain the following analogue of Theorem 2.4.

Proposition 4.2. Suppose the assumptions of Theorem 4.1 are satisfied. Assume moreover that $v^{1-p^{\prime}} \notin L^{1}\left(\mathbb{R}^{n}\right)$. Then, for each fixed $f \in L_{v}^{p}$, and any sequence $f_{m} \in L_{c, 0}^{\infty}$ approximating $f$ in the $L_{v}^{p}$ norm,

(i) $\mathscr{F} f(\gamma)=\lim \hat{f}_{m}(\gamma)$ at every $\gamma \in \mathbb{R}^{n}$;

(ii) $\mathscr{F} f=\lim _{m} \hat{f}_{m}$ in the $L^{q}\left(S_{n-1}(\rho)\right)$ norm.

Note that (ii) in particular means that $\mathscr{F}: L_{v}^{p} \rightarrow L^{q}\left(S_{n-1}(\rho)\right)$ is the bounded extension of $f \rightarrow \hat{f}: L_{c, 0}^{\infty} \rightarrow L^{q}\left(S_{n-1}(\rho)\right)$.

As a corollary of Theorem 4.1, we obtain the following restriction property for power weights. 
Corollary 4.3. Let $1<p<\infty, 0<q<\infty$. Suppose $v(x)=|x|^{\beta p}$ and $n / p^{\prime}<\beta<\left(n / p^{\prime}\right)+1$. Then, for each $\rho>0$,

$$
\|\mathscr{F} f\|_{L_{S_{n-1}(\rho)}^{q}} \leq L(\rho, v)\|f\|_{p, v}
$$

for all $f \in L_{v}^{p}$, where

$$
\begin{aligned}
L(\rho, v)= & 2|S|^{1 / p^{\prime}+1 / q} \cdot \pi^{\beta-n / p^{\prime}} \rho^{(n-1) / q-n / p^{\prime}+\beta} \\
& \times\left[\left(n+p^{\prime}-\beta p^{\prime}\right)^{-1}+\left(\beta p^{\prime}-n\right)^{-1}\right]^{1 / p^{\prime}} .
\end{aligned}
$$

Proof. Using Remark 1.1.2, we see that the constant $K(\rho, v)$ in Theorem 4.1 is

$$
K(\rho, v)=2|S|^{1 / p^{\prime}}\left[(\pi \rho)^{p^{\prime}} \int_{0}^{1 / \pi \rho} r^{n-1+p^{\prime}-\beta p^{\prime}} d r+\int_{1 / \pi \rho}^{\infty} r^{n-1-\beta p^{\prime}} d r\right]^{1 / p^{\prime}}
$$

when $v(x)=|x|^{\beta p}$. The result follows.

Remark 4.4. In the above corollary, the lower bound $\beta=n / p^{\prime}$ of the range of $\beta$ is excluded. This value corresponds to the weight $v(x)=|x|^{n(p-1)}$ which is precisely the one for which we proved that no restriction property can hold for all $f \in L_{c, 0}^{\infty}$ (cf. Corollary 3.2). Further comparisons between restriction properties are discussed in the next subsection.

4.2. Comparison of circle restriction properties in the power weight case. Suppose $n=2$. We wish to compare Corollary 4.3 above with the circle restriction property contained in [5, Corollary 2]. To that purpose, we remark the following particular facts:

A. In [5], estimates of the form

$$
\|\hat{f}\|_{L^{q}\left(S_{1}(\rho)\right)} \leq C \cdot \rho^{1 / q-2 / p^{\prime}+\beta}\|f\|_{p, v_{1}}
$$

are proved for each $\rho>0$, under the assumptions that $(p, q)$ belongs to a certain range $E$ and $v_{1}=|x|^{\beta p}$ with $0 \leq \beta_{0}(p, q) \leq \beta<2 / p^{\prime}$. (For the explicit definition of $E$ and $\beta_{0}(p, q)$, see [5, Corollary 2].)

In fact, (4.4) is initially proved on a dense subspace of simple functions which includes $L_{c}^{\infty}$. The inequality is then extended to the whole of $L_{v_{1}}^{p}$ by a norm limit process in which $\hat{f}$ is defined by Cauchy sequences in the $L^{q}\left(S_{1}(\rho)\right)$ norm.

Note also that the classical restriction theorem of Zygmund [11] is a particular case of (4.4) ( $\beta$ is then zero).

In order to compare (4.4) with some results of this paper, we remark that $v_{1}(x)^{1-p^{\prime}}=|x|^{-\beta p^{\prime}}$ is not integrable at infinity for $\beta<2 / p^{\prime}$. Lemma 2.2 thus implies that $L_{c, 0}^{\infty}$ is dense in $L_{v_{1}}^{p}$. Therefore, for each $\rho>0$ and for each fixed $f \in L_{v_{1}}^{p}$,

$$
\hat{f}=\lim _{m} \hat{f}_{m} \text { in the } L^{q}\left(S_{1}(\rho)\right) \text { norm }
$$

for any sequence $f_{m} \in L_{c, 0}^{\infty}$ approximating $f$ in the $L_{v_{1}}^{p}$ norm.

Note also that Lemma 1.1.1 is not applicable to $L_{v_{1}}^{p}$ and, as a consequence, we do not have any general conclusion as regards the pointwise everywhere existence of $\mathscr{F} f(\gamma)$ when $f \in L_{v_{1}}^{p} \backslash\left(L^{1} \cap L_{v_{1}}^{p}\right)$. 
B. On the other hand, Corollary 4.3 in particular implies that under the assumptions $n=2,1<p<\infty, 0<q<\infty, v_{2}(x)=|x|^{\beta p}$, and $2 / p^{\prime}<\beta<2 / p^{\prime}+1$, the following estimate holds for each $\rho>0$ and for all functions in $L_{c}^{\infty}$ :

$$
\|\hat{f}-\hat{f}(0)\|_{L^{q}\left(S_{1}(\rho)\right)} \leq C \rho^{1 / q-2 / p^{\prime}+\beta}\|f\|_{p, v_{2}} .
$$

C. As a consequence of $A$ and $B$, estimates (4.4) and (4.6) both hold on the common moment subspace $L_{c, 0}^{\infty}$ of $L_{v_{1}}^{p}$ and $L_{v_{2}}^{p}$. The function in the left side is then the classical Fourier transform $\hat{f}$ in both inequalities.

When $f \in L_{c}^{\infty} \backslash L_{c, 0}^{\infty}$, these estimates also hold but the function in the left side is either $\hat{f}$ or $\hat{f}-\hat{f}(0)$ according as $\beta<2 / p^{\prime}$ or $\beta>2 / p^{\prime}$. A fortiori, the bounded extensions of $f \rightarrow \hat{f}: L_{c, 0}^{\infty} \rightarrow L^{q}\left(S_{1}(\rho)\right)$ to the respective domains $L_{v_{1}}^{p}, L_{v_{2}}^{p}$ differ accordingly. When $\beta<2 / p^{\prime}$, the extension is defined by (4.5). When $\beta>2 / p^{\prime}$, Proposition 4.2 shows that the bounded extension is $\mathscr{F} f$.

D. Remark $C$ naturally raises the question of knowing what happens when $n=2, \beta=2 / p^{\prime}$. This question is answered in $\S 3$. In fact, Corollary 3.2 in particular implies that for $n=2,(p, q) \in E, \beta=2 / p^{\prime}$, neither (4.4) nor (4.6) can hold for all $f \in L_{c}^{\infty}$.

\section{FURTHER CONDITIONS FOR THE BOUNDEDNESS OF $\mathscr{F}$}

Using the pointwise estimate of $\mathscr{F} f(\gamma)$ given by Lemma 1.1.1, we easily obtain the following general sufficient condition in order that $\mathscr{F}$ be bounded from $L_{v}^{p}$ into $L_{u}^{q}$.

Theorem 5.1. Let $1<p<\infty, 0<q<\infty, v \in L_{\text {loc }}^{1}\left(\mathbb{R}^{n}\right), v>0$ a.e. Assume $|t|^{p^{\prime}} v^{1-p^{\prime}} \in L_{\mathrm{loc}}^{1}\left(\mathbb{R}^{n}\right), v^{1-p^{\prime}} \in L^{1}\left(B_{a}^{c}\right)$ for some $a>0$. Suppose $u$ is nonnegative, $u \in L_{\text {loc }}^{1}\left(\mathbb{R}^{n} \backslash\{0\}\right)$, and

$$
K(\rho, v)=2\left\{(\pi \rho)^{p^{\prime}} \int_{|t|<1 /(\pi \rho)}|t|^{p^{\prime}} v^{1-p^{\prime}} d t+\int_{|t|>1 /(\pi \rho)} v^{1-p^{\prime}} d t\right\}^{1 / p^{\prime}} .
$$

If $D=\left(\int_{\mathbb{R}^{n}} K(|\gamma|, v)^{q} u(\gamma) d \gamma\right)^{1 / q}<\infty$, then $\|\mathscr{F} f\|_{q, u} \leq D\|f\|_{p, v}$ for all $f \in$ $L_{v}^{p}$.

A similar statement holds when $u$ is replaced by a (possibly) nonradial positive measure on $\mathbb{R}^{n}$ :

Theorem 5.2. Suppose $p, q, v$ are as in Theorem 5.1 and $\mu \in M_{+}\left(\mathbb{R}^{n}\right)$. If

$$
D^{*}=\left(\int_{\mathbb{R}^{n}} K(|\gamma|, v)^{q} d \mu(\gamma)\right)^{1 / q}<\infty,
$$

then $\|\mathscr{F} f\|_{q, \mu} \leq D^{*}\|f\|_{p, v}$ for all $f \in L_{v}^{p}$.

Remark 5.3. 1. For $\mu=\mu_{\rho}$, Theorem 5.2 reduces to Theorem 4.1. If, in addition $1<p \leq q<\infty$, this theorem is equivalent to Theorem 1.2 as concerns the class of weights for which an estimate of the form $\|\mathscr{F} f\|_{q, \mu_{\rho}} \leq C s t\|f\|_{p, v}$ holds. The constant in Theorem 5.2 is nevertheless sharper.

In other words, $D^{*}<\infty$ if and only if $A_{1}^{*}<\infty$ and $A_{2}^{*}<\infty$. Moreover, $D^{*}<C^{*}$ (for the values of $A_{1}^{*}, A_{2}^{*}$, and $C^{*}$, see the statement of Theorem $1.2 \mathrm{a})$. 
2. For $1<p \leq q<\infty$, Theorem 5.1 is weaker then Theorem $1.1 \mathrm{a}^{\prime}$, that is:

(a) $D<\infty$ implies $A_{1}<\infty, A_{2}<\infty$.

(b) There exist $u, v$ such that $A_{1}<\infty, A_{2}<\infty$, and $D=+\infty$.

Proof. (a) We first remark that for any $p, q$ such that $0<q<\infty, 1<p<\infty$, condition $D<\infty$ implies $A_{1}<\infty, A_{2}<\infty$. This follows from the following majorization, for each $y>0$,

$$
\begin{aligned}
A_{1}(y)^{q} & =\left(\int_{|x|<y}|x|^{q} u(x / \pi) d x\right)\left(\int_{|x|<1 / y}|x|^{p^{\prime}} v^{1-p^{\prime}} d x\right)^{q / p^{\prime}} \\
& \leq \int_{|x|<y} u(x / \pi)\left(|x|^{p^{\prime}} \int_{|t|<1 /|x|}|t|^{p^{\prime}} v^{1-p^{\prime}} d t\right)^{q / p^{\prime}} d x \\
& \leq \int_{\mathbb{R}^{n}} u(x / \pi)(\cdots)^{q / p^{\prime}} d x,
\end{aligned}
$$

and a similar one corresponding to $A_{2}$. These imply $A_{1}^{q}+A_{2}^{q} \leq c D^{q}$, hence assertion (a).

(b) Consider $u(x)=|x|^{\alpha}, v(x)=|x|^{\beta}$. These weights satisfy $A_{1}<\infty$, $A_{2}<\infty$ if $-n-q<\alpha<-n, n / p^{\prime}<\beta / p<1+n / p^{\prime}$, and $n(1 / q+1 / p-1)+$ $\alpha / q+\beta / p=0$.

Since the last equality implies $\rho^{n-1} u(\rho) K(\rho, v)^{q}=1 / \rho$, we obtain $D=+\infty$ for the corresponding weights. This proves (b).

3. If $0<q<p<\infty, p>1$, Theorem 1.1b includes Theorem 5.1, i.e., $D<\infty$ implies $B_{1}<\infty, B_{2}<\infty$.

Proof. Since $r / q-1=r / p$, the constant $B_{1}$ of Theorem $1.1 \mathrm{~b}$ is

$$
\begin{aligned}
B_{1}= & \int_{\mathbb{R}^{n}}\left(\int_{|x| \leq 1 /|y|} u(x / \pi)|x|^{q} d x\right)\left(\int_{|x| \leq 1 /|y|} u(x / \pi) \cdot|x|^{q} d x\right)^{r / p} \\
& \cdot\left(\int_{|x| \leq|y|}|x|^{p^{\prime}} v^{1-p^{\prime}} d x\right)^{r / q^{\prime}}|y|^{p^{\prime}} v(y)^{1-p^{\prime}} d y .
\end{aligned}
$$

But $D<\infty$ implies $A_{1}<\infty$. Therefore, the second factor inside the integral over $\mathbb{R}^{n}$ is majorized by $c\left(\int_{|x| \leq|y|}|x|^{p^{\prime}} v^{1-p^{\prime}} d x\right)^{-q r / p^{\prime} p}$.

Since $-q r / p p^{\prime}+r / q^{\prime}=q / p^{\prime}-1$, one obtains

$$
B_{1} \leq c \lim _{\substack{\varepsilon \rightarrow 0+\\ R \rightarrow+\infty}} \int_{\varepsilon}^{R} I(1 / \rho)\left\{\int_{0}^{\rho} s^{n-1+p^{\prime}} V(s) d s\right\}^{q / p^{\prime}-1} \rho^{n-1+p^{\prime}} V(\rho) d \rho,
$$

where

$$
I(t)=\int_{0}^{t} s^{n-1+q} \int_{S} u(s \omega / \pi) d \sigma d s, \quad \text { and } \quad V(\rho)=\int_{S} v(\rho \omega)^{1-p^{\prime}} d \sigma .
$$

Integrating by parts and using again $A_{1}<\infty$, one obtains

$$
B_{1} \leq c+\left.\left.c^{\prime} \int_{\mathbb{R}^{n}}|\gamma|^{q} u(\gamma / \pi)\left|\int_{|x| \leq 1 /|\gamma|}\right| x\right|^{p^{\prime}} v^{1-p^{\prime}} d x\right|^{q / p^{\prime}} d \gamma .
$$


Proceeding in the same way for estimating $B_{2}$, one finally gets $B_{1}+B_{2} \leq$ $c_{1}+c_{2} D^{q}$, which yields assertion 3 .

\section{ACKNOWLEDGMENT}

We wish to express our gratitude to Professor H. P. Heining for fruitful discussions which led to several improvements of our manuscript. We are also grateful to Professor J. J. Benedetto for his helpful comments on some parts of our work.

\section{REFERENCES}

1. K. F. Andersen and B. Muckenhoupt, Weighted weak type Hardy inequalities with applications to Hilbert transforms and maximal functions, Studia Math. 72 (1982), 9-26.

2. J. J. Benedetto and H. P. Heinig, Fourier inequalities with measure weights, Adv. in Math. (to appear).

3. __ Fourier inequalities with measure weights. II, Proc. 2nd Internat. Conf. on Function Spaces, Poznan 1989, Teubner Texte zur Math., Teubner, Leipzig (to appear).

4. J. S. Bradley, Hardy inequalities with mixed norms, Canad. Math. Bull. 21 (1978), 405-408.

5. C. Carton-Lebrun and H. P. Heinig, Weighted extensions of restriction theorems for the Fourier transform, Recent Advances in Fourier Analysis and its Applications, NATO Adv. Sci. Inst. Ser. C, 315, Kluwer Academic, 1990, pp. 579-596.

6. W. Maz'ja, Einbettungssätze für Sobolevsche Räume, Teil 1, Teubner Texte zur Math., Teubner, Leipzig, 1989.

7. B. Muckenhoupt, R. L. Wheeden, and W.-S. Young, $L^{2}$ multipliers with power weights, Adv. in Math. 49 (1983), 170-216.

8. C. Sadosky and R. L. Wheeden, Some weighted norm inequalities for the Fourier transform of functions with vanishing moments, Trans. Amer. Math. Soc. 300 (1987), 521-533.

9. E. T. Sawyer, Weighted Lebesgue and Lorentz norm inequalities for the Hardy operator, Trans. Amer. Math. Soc. 281(1984), 329-337.

10. G. J. Sinnamon, Operators on Lebesgue spaces with general measures, Ph.D. Thesis, McMaster Univ., Hamilton, Canada, 1987.

11. A. Zygmund, On Fourier coefficients and transforms of functions of two variables, Studia Math. 50 (1974), 189-201.

Department of Mathematics, University of Mons, 7000 Mons, Belgium

E-mail address: mlebrun@bmsuem11.bitnet 